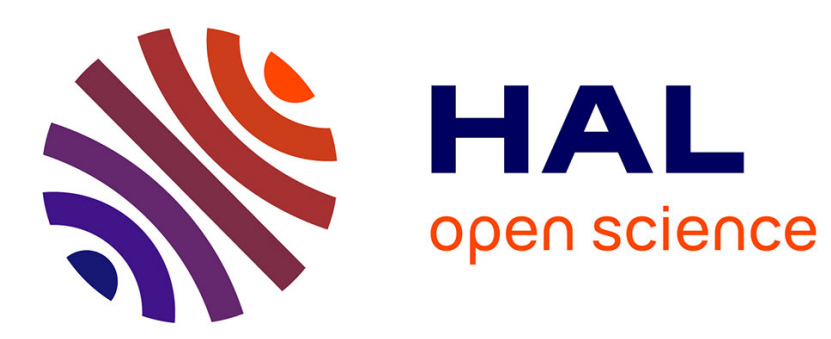

\title{
Critical dimension and 1/D expansion for the random anisotropy axis model
}

\author{
N. Sourlas
}

\section{To cite this version:}

N. Sourlas. Critical dimension and 1/D expansion for the random anisotropy axis model. Journal de Physique Lettres, 1981, 42 (11), pp.233-236. 10.1051/jphyslet:019810042011023300 . jpa-00231916

\section{HAL Id: jpa-00231916 https://hal.science/jpa-00231916}

Submitted on 1 Jan 1981

HAL is a multi-disciplinary open access archive for the deposit and dissemination of scientific research documents, whether they are published or not. The documents may come from teaching and research institutions in France or abroad, or from public or private research centers.
L'archive ouverte pluridisciplinaire HAL, est destinée au dépôt et à la diffusion de documents scientifiques de niveau recherche, publiés ou non, émanant des établissements d'enseignement et de recherche français ou étrangers, des laboratoires publics ou privés. 


\title{
Critical dimension and 1/D expansion for the random anisotropy axis model
}

\author{
N. Sourlas \\ Laboratoire de Physique Théorique de l'Ecole Normale Supérieure (*), 24, rue Lhomond, 75231 Paris Cedex 05, France \\ (Reçu le 14 octobre 1980, révisé le 7 avril 1981, accepté le 8 avril 1981)
}

\begin{abstract}
Résumé. - Nous montrons que le modèle de matériaux magnétiques amorphes où l'axe d'anisotropie est aléatoire a comme dimension critique supérieure $D=4$. Nous étudions par la suite la transition de phase dans ce modèle dans le cadre du développement en puissances de $1 / D$. Tandis que le terme dominant quand $D \rightarrow \infty$ ne dépend pas du couplage avec l'axe d'anisotropie, les corrections en $1 / D$ et $1 / D^{2}$ décroissent la température de transition entre la phase désordonnée et la phase à ordre ferromagnétique.
\end{abstract}

\begin{abstract}
We show that the upper critical dimension for the random anisotropy model of amorphous magnets is four as in the case of the pure ferromagnetic model. We then study the phase transition in this model in the framework of the $1 / D$ expansion (where $D$ is the dimension of space). While the leading term for $D \rightarrow \infty$ does not depend on the anisotropy coupling, the $1 / D$ and $1 / D^{2}$ corrections decrease the transition temperature between the disordered and the ferromagnetic phase.
\end{abstract}

A lot of work has recently been devoted to the random anisotropy model (RAM) introduced by Harris, Plischke and Zuckermann [1] for the description of the magnetic properties of certain amorphous intermetallic compounds. Mean-field approximation techniques and renormalization group inspired calculations as well as numerical simulations have been performed.

Jayaprakash and Kirkpatrick [2] carried out the most extensive Monte-Carlo simulations in the Isinglike limit of infinite anisotropy energy, in three dimensions. They find that the ground state of this model exhibits short-range magnetic order and no long-range order but they cannot determine whether a sharp phase transition occurs.

Derrida and Vannimenus [3] have studied the infinite range version of this model. For conventional spin systems the infinite range approximation is equivalent to the mean-field approximation. They find a second order transition from the high temperature phase to a ferromagnetic phase, and no sign of a spin-glass phase. The transition temperature does not depend on the anisotropy energy and is the same as in the pure ferromagnetic model.

Aharony has investigated the critical properties of this model. Using the $\varepsilon=4-D$ expansion, and starting from the disordered phase, he found no

(*) Laboratoire propre du C.N.R.S. associé à l'Ecole Normale Supérieure et à l'Université de Paris-Sud. stable fixed point at order $\varepsilon$. Pelcovits, Pytte and Rudnick [5] argue that, at low temperature, there exists an Edwards-Anderson spin-glass phase in this model, and that this is the origin of the instability of the fixed point, found by Aharony. They use a low temperature (spin wave) renormalization group calculation and treat the anisotropy coupling $c$ (see below) as a first order perturbation.

Although it is very probable that a spin-glass phase exists in this model at low temperature, nobody, up to now, succeeded in finding such a phase by a satisfactory mean-field treatment and then studying its critical properties.

In this note we will argue that a lot of care is necessary when treating the anisotropy energy as a first order perturbation and that a further analysis is needed.

Then we will study the phase transition in the large $D$ approximation (where $D$ is the dimensionality of space) keeping $\beta D$ fixed and taking the anisotropy coupling $c$ to all orders into account. For conventional spin systems the $D \sim$ infinite approximation [8] is equivalent to the mean-field approximation and the expansion in powers of $1 / D$ can be considered as a systematic way of computing corrections to the mean-field approximation. Keeping only the leading term in the $D \rightarrow \infty$ limit we will recover the results of Derrida and Vannimenus [3]. (This was not obvious in advance, because the equivalence of the large $D$ approximation with the infi- 
nite range approximation has not been demonstrated for quenched systems.) But, contrary to the leading term, the $1 / D$ and $1 / D^{2}$ corrections to the transition temperature, will depend on the anisotropy coupling. As a result of this at large but finite dimensions the transition temperature will be lower than in the pure ferromagnetic model.

We start by recalling the Harris, Plischke and Zuckermann Hamiltonian :

$$
H / k T=\beta \sum_{\langle i j\rangle} \sigma_{i} \sigma_{j}+c \sum_{i}\left(\sigma_{i} \eta_{i}\right)^{2}
$$

where the sum $\sum_{\langle i j\rangle}$ extends to all nearest neighbour pairs on a $D$-dimensional cubic lattice, $\boldsymbol{\sigma}_{\boldsymbol{i}}$ and $\boldsymbol{\eta}_{i}$ are $N$-dimensional unit vectors and $\eta_{i}$ is randomly oriented. (We assume a spatially uncorrelated and isotropic on the unit sphere distribution of the $\eta_{i}{ }^{\circ}$ s.)

The upper critical dimension is determined by the strongest infrared singularities. In order to make the discussion clearer we shall compare the infrared behaviour of this model with the infrared behaviour of the random field model, for which it is known that the upper critical dimension is six $[6,7]$. A first remark is that in the latter case there is a hidden supersymmetry [7] which is responsible for this shift of the critical dimensionality from four to six and that there is no such supersymmetry for the random anisotropy model.

It is convenient to introduce the Lagrangian of the corresponding field theory for the two models

$$
\begin{aligned}
& \mathcal{L}_{\mathrm{r} . \mathrm{f}}(x)=\frac{1}{2} \phi\left(-\nabla+m^{2}\right) \phi+\lambda\left(\phi^{2}\right)^{2}+\mathbf{h} \varphi \\
& \mathcal{L}_{\mathrm{r} . \mathrm{a}}(x)=\frac{1}{2} \phi\left(-\nabla+m^{2}\right) \phi+\lambda\left(\phi^{2}\right)^{2}+c(\eta \phi)^{2}
\end{aligned}
$$

where $\mathbf{h}(x)$ is a random Gaussian field and $\eta(x)$ is a random direction $\left(\boldsymbol{\eta}^{2}=1\right)$.

In order to compute the correlation functions, we first calculate the connected Feynman diagrams in the perturbation expansion in powers of $\lambda$ and $\mathbf{h}$ for the random field model, in powers of $\lambda$ and $c$ for the random anisotropy model. Then we average over the random field $h(x)$ or the random axis $\eta(x)$.

Figure 1 shows second order in $\lambda$ Feynman diagrams contributing to the self-energy before averaging over h or over $\eta$. Diagrams (a) and (b) contribute to the self-energy in the random field model while the diagrams (c), (d), (e) and (f) are for the random anisotropy model. Figure 2 shows the diagrams (a), (b), (c), (d) after the $h$ or $\eta$ averaging.

The shift in the critical dimensionality of the random field model comes from the additional propagators appearing in the diagrams (a) and (b).

To first order in the anisotropy coupling $c$ there is no difference between the two models. The important difference comes from diagrams like (e) and (f) which have been previously ignored. They do not contribute to the random field model because they are disconnected before the $\mathbf{h}$ averaging but they do

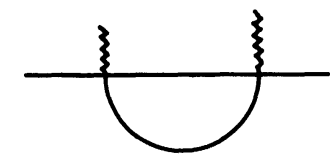

(a)

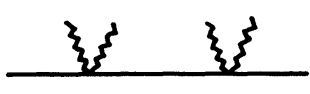

(b)

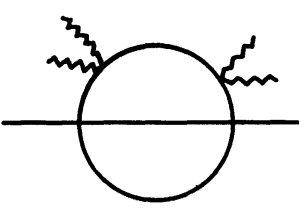

(e)

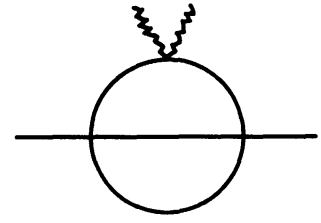

(c)

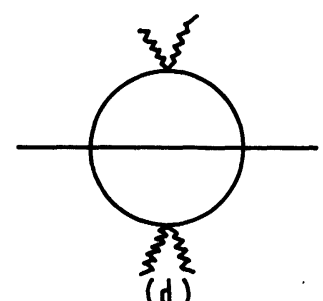

(d)

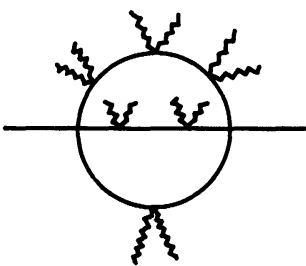

(f)
Fig. 1. - Self-energy diagrams before averaging over the random field (diagrams (a) and (b)) or random axis (diagrams (c) to (f)) distribution.

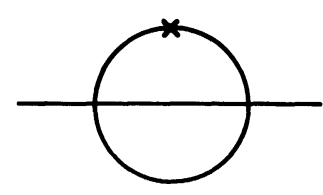

(a)

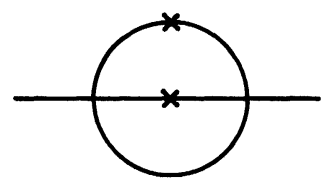

(b)

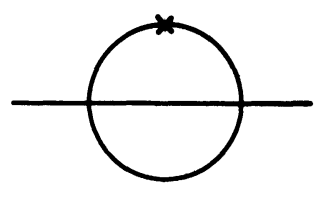

(c)

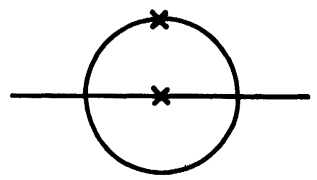

(d)

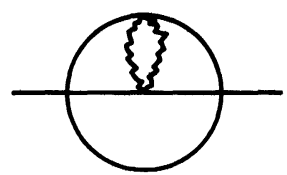

(d')

Fig. 2. - The same diagrams (a) to (d) as in figure 1 after averaging over the random field or random axis distribution.

contribute to the random anisotropy model. They are more and more infrared divergent for $m^{2} \rightarrow 0$ as one goes to higher orders in $c$. In fact those divergences are spurious because they sum up in order to renormalize the mass. One should expand the mass in powers of $\lambda$ and $c$ :

$$
m^{2}=\sum_{i, j=0}^{\infty} m_{i j} \lambda^{i} c^{j}
$$


The constants $m_{i j}$ should be determined by imposing the condition :

$$
\Gamma^{(2)}\left(p^{2}=m^{2}\right)=0
$$

at every order in $\lambda$ and $c$ (where $\Gamma^{(2)}$ is the Fourier transform of the 2-point function). Because of this condition, the counterterms proportional to $m_{i j}$ will cancel all additional infrared divergences and the power counting will be the same as in the absence of the anisotropy. So the upper critical dimension of this model is four as for the pure ferromagnetic model. This resummation mechanism does not work for the random field model because in this case the corresponding diagrams are disconnected and therefore absent. The crucial importance of the condition (5) for the correct treatment of the infrared divergences has already been emphasized by Kinoshita [9] in his study of the infrared behaviour of quantum electrodynamics. Another way of phrasing the same result is to say that $(\phi \eta)^{2}$ is quadratic in $\phi$ and is therefore a relevant operator in the renormalization group terminology and it is incorrect to simply treat this term as a perturbation, as in the case of a marginal operator.

We now proceed to the $1 / D$ expansion. This expansion has been introduced for the Ising model by Fisher and Gaunt, and Englert [8]. A most elegant application of the $1 / D$ expansion to the $0(n)$ Heisenberg model has been carried out recently by Balian, Drouffe and Itzykson [10]. We will closely follow the method of reference [10].

This method has also been generalized and applied to the case of lattice gauge theories [11].

We start from the high temperature expansion for the free energy. This can most easily be generated once the "cumulants" are known. We introduce the generating function for the cumulants :

$$
\begin{aligned}
u^{\prime}(\boldsymbol{\phi}, \boldsymbol{\eta}) & =\log \int \mathrm{d} \boldsymbol{\sigma} \mathrm{e}^{c(\boldsymbol{\sigma} \boldsymbol{\eta})^{2}+\phi \boldsymbol{\sigma}} \\
& =\sum_{n} \frac{1}{n !} u_{\alpha_{1} \ldots \alpha_{n}}^{(n)} \phi^{\alpha_{1}} \ldots \phi^{\alpha_{n}} .
\end{aligned}
$$

In order to average the free energy over the $\eta$ distribution, it is sufficient to only average the cumulants. So we next introduce the generating function of the averaged cumulants :

$$
\begin{aligned}
u(\phi) & =\int \mathrm{d} \eta \log \int \mathrm{d} \sigma \mathrm{e}^{c(\boldsymbol{\sigma} \eta)^{2}+\phi \sigma} \\
& =\sum_{n} \frac{1}{n !} \phi^{\alpha_{1}} \ldots \phi^{\alpha_{n}} \int \mathrm{d} \eta u_{\alpha_{1} \ldots \alpha_{n}}^{(n)} .
\end{aligned}
$$

In this way, we treat exactly the anisotropy energy, by incorporating it into the measure. We compute the free energy $F(\beta, h)$ in the presence of an external field $\mathbf{h}$. We make the approximation of keeping a finite number of high temperature one line irredu- cible diagrams, and take the Legendre transform. The dimensionality $D$ of the space enters in the coefficients of the different diagrams. The Legendre transformation can be formulated as a stationarity requirement for the free energy with respect to magnetization $\mathbf{m}$.

For $\beta<\beta_{\mathrm{c}}, F$ becomes stationary for $\mathbf{m}=0$. At $\beta=\beta_{\mathrm{c}},\left.\frac{\partial F}{\partial \mathbf{m}^{2}}\right|_{\mathbf{m}^{2}=0}$ changes sign.

This is the way the critical temperature $\beta_{\mathrm{c}}$ is computed, and the result is expanded in powers of $1 / D$. It can be shown that keeping a finite number of diagrams is equivalent to keeping a finite number of terms in the $1 / D$ expansion. The order in $1 / D$ in which one works uniquely determines the high temperature diagrams one has to compute. A more detailed description of this prescription and its justification can be found in reference [10] together with detailed references to the original work.

As $\mathbf{m}(x)$ can in general be position dependent, the stationarity requirement for the free energy is a functional equation. This equation is very difficult to treat and one makes the simple ansatz that the stationary configuration is translation invariant, $\mathbf{m}(x)=\mathbf{m}$.

Because of this ansatz, we can also use in the variational calculation the averaged cumulants given by equation (7) instead of those given by equation (6). In our case, this ansatz of a translation invariant configuration neglects possible local correlations between $m(x)$ and $\eta(x)$. For this reason it is not possible to see a spin-glass phase in this way.

.It is easy to show that the zeroth order in $\beta$ contribution to $\left.\frac{\partial F}{\partial \mathbf{m}^{2}}\right|_{\mathbf{m}^{2}=0},\left(\right.$ that is $\left.\left.\frac{\partial u}{\partial \phi^{2}}\right|_{\phi^{2}=0}\right)$, does not depend on $c$. As this term is the only contribution in the $D \rightarrow \infty$ limit, we see that for $D \rightarrow \infty$ the critical temperature is the same as in the absence of anisotropy, in agreement with the result of Derrida and Vannimenus [3].

For $c=0$ the result is known from reference [10]. For general $c \neq 0$, we were unable to compute the analytic form of the cumulants. It is only when $c \gg 1$ that we have the following analytic form :

$$
\begin{aligned}
u\left(\phi^{2}, c=\infty\right) & =\frac{\phi^{2}}{2} \frac{1}{N}-\left(\frac{\phi^{2}}{2}\right)^{2} \frac{1}{N(N+2)}+ \\
& +\left(\frac{\phi^{2}}{2}\right)^{3} \frac{8}{3} \frac{1}{N(N+2)(N+4)}+\cdots .
\end{aligned}
$$

In the absence of anisotropy we have [10]

$$
\begin{aligned}
& u\left(\phi^{2}, c=0\right)=\frac{\phi^{2}}{2} \frac{1}{N}-\left(\frac{\phi^{2}}{2}\right)^{2} \frac{1}{N^{2}(N+1)}+ \\
&+\left(\frac{\phi^{2}}{2}\right)^{3} \frac{8}{3} \frac{1}{N^{3}(N+2)(N+4)}+\cdots
\end{aligned}
$$

(we recall that we are considering the $0(N)$ symmetric model). 
We see that the higher cumulants are larger for $c=\infty$ than for $c=0$ and that the first cumulant remains unchanged. This is the reason why the $1 / D$ corrections are much larger in the presence of anisotropy than in the pure ferromagnetic model (particularly for large $N$ ) while in the mean-field approximation those models share the same behaviour.

Table I shows the diagrams we have computed and their contribution to $\left.\frac{\partial F}{\partial \mathbf{m}^{2}}\right|_{\mathbf{m}^{2}=0}$.

Table I. - High temperature diagrams and their contribution to $\left.\frac{\partial F}{\partial m^{2}}\right|_{m^{2}=0}$

\begin{tabular}{cc}
$\begin{array}{cc}c=\infty \\
\frac{1}{N}\end{array}$ & $\begin{array}{c}c=0 \\
N\end{array}$ \\
$-\frac{2 \beta^{2} D}{N^{2}}$ & $-\frac{2 \beta^{2} D}{N^{3}}$ \\
$\frac{4 \beta^{3} D}{N^{2}(N+2)}$ & $\frac{4 \beta^{3} D}{N^{4}(N+2)}$ \\
$-\frac{6 \beta^{4} D(2 D-1)}{N^{4}}$ & $\frac{12 \beta^{4} D^{2}}{N^{5}}$ \\
\hline$-\frac{6 \beta^{4} D(2 D-1)}{N^{5}}$
\end{tabular}

The critical values $\beta_{\mathrm{c} \infty}$ and $\beta_{\mathrm{c} 0}$ (for $c=\infty$ and $c=0$ respectively) of $\beta$ for which the phase transition occurs are given by

$$
\begin{aligned}
& \left(\frac{2 \beta_{\mathrm{c} 0} D}{N}\right)^{-1}=1-\frac{1}{2 D}-\frac{2-2 /(N+2)}{(2 D)^{2}} \\
& \left(\frac{2 \beta_{\mathrm{c} \infty} D}{N}\right)^{-1}=1-\frac{N}{2 D}+\frac{N(N-2)(N+3)}{(2 D)^{2}(N+2)} .
\end{aligned}
$$

For the particular case $N=2$ we have computed the cumulants numerically and then the critical temperature $\beta_{\mathrm{c}}$ (in the $1 / D$ expansion) for several values of the anisotropy coupling $c$. We found a smooth variation of the results from $c=0$ to $c=\infty$ and nothing particular is happening at intermediate values of $c$.

From equation (11) we see that for large anisotropy coupling, the $1 / D$ corrections increase dramatically with $N$ and therefore the $1 / D$ expansion is good only for small $N$. While for the pure ferromagnetic case the $1 / N$ and $1 / D$ expansions seem to commute, this is certainly not the case for large $c$. The presence of the anisotropy coupling has a tendency to decrease the critical temperature $T_{\mathrm{c}} \sim 1 / \beta_{\mathrm{c}}$ where ferromagnetic order appears, in agreement with our intuitive expectations.

In the previous analysis we examined the case of a second order transition. It is also possible to search for a first order transition. For this purpose one should look for stationarity points of the free energy with respect to finite (different from zero) values of the magnetization. (In the previous analysis we examined only the neighbourhood of $m=0$.) We were unable to do this investigation analytically. We carried it out numerically only in the particular case of $N=2$ and no sign of a first order transition was found.

Finally in all the previous analysis we have assumed translational invariance for the stationary configuration. In their numerical simulations (in three dimensions and for large anisotropy coupling ${ }^{~} c$ ), Jayaprakash and Kirkpatrick [2] found evidence, of the existence of short-range order and absence of long-range order at very low temperature. Our analysis shows that such a phase does not exist at very large dimension (when the $1 / D$ corrections are neglected, there is no dependence on the anisotropy coupling $c$ ). It is very difficult (within the framework of our method) to look for such non translational invariant configurations for finite $D$ (including the $1 / D$ corrections).

Acknowledgments. - It is a pleasure to thank S. Kirkpatrick and J. Vannimenus for calling my attention to the problem of the critical dimensionality of the random axis model and for stimulating discussions. I also thank E. Brezin for useful discussions.

\section{References}

[1] Harris, R., Plischke, M., Zuckermann, M. J., Phys. Rev. Lett. 31 (1973) 160.

[2] Jayaprakash, C., Kirkpatrick, S., Phys. Rev. B 21 (1980) 4072.

[3] Derrida, B., Vannimenus, J., J. Phys. C 13 (1980) 3261.

[4] Aharony, A., Phys. Rev. B 12 (1975) 1038.

[5] Pelcovits, R. A., Pytte, E., Rudnick, J., Phys. Rev. Lett. 40 (1978) 476 ;

Pelcovits, R. A., Phys. Rev. B 19 (1979) 465 ;

Pytte, E., Phys. Rev. B 18 (1978) 5046.

[6] Imry, Y., Ma, S., Phys. Rev. Lett. 35 (1975) 1399.
[7] Parisi, G., Sourlas, N., Phys. Rev. Lett. 43 (1979) 744.

[8] Englert, F., Phys. Rev. 129 (1963) 567;

Fisher, M. E., Gaunt, D. S., Phys. Rev. 133 (1964) A 224.

[9] Kinoshita, T., J. Math. Phys. 3 (1962) 650.

[10] Balian, R., Drouffe, J. M., Itzykson, C., Phys. Rev. D 19 (1975) 2514.

[11] Drouffe, J. M., Parisi, A., Sourlas, N., Nucl. Phys. B 161 (1979) 397 ;

Drouffe, J. M., to appear in the Proceedings of Common trends in particle and condensed matter physics (Les Houches, Feb. 1980). 